\title{
Pancoast tumor resection with preservation of brachial plexus and hand function
}

\author{
Gavin A. Davis, M.B.B.S., F.R.A.C.S., ${ }^{1,2}$ AND Simon KNight, M.B.B.S., F.R.A.C.S. ${ }^{3}$ \\ ${ }^{1}$ Department of Neurosurgery, Cabrini Hospital; ${ }^{2}$ Department of Neurosurgery, Austin \\ Hospital; and ${ }^{3}$ Department of Thoracic Surgery, Austin Hospital, Victoria, Australia
}

\begin{abstract}
Object. Pancoast tumors are aggressive bronchogenic lesions of the lung apex that are rapidly fatal if untreated. Modern treatment includes induction chemotherapy and radiotherapy prior to resection, but many authors also resect the T- 1 nerve root (with or without the C- 8 nerve root and the lower trunk of the brachial plexus) as part of the therapy, causing significant loss of hand function in many patients. The current authors determined whether a different approach allowing preservation of the brachial plexus and hand function could be adopted without compromising patient survival. An extensive historical review of Pancoast tumors is presented as a baseline for clinical comparison.

Methods. Five patients harboring Pancoast tumors with brachial plexus involvement underwent surgery performed by both a neurosurgeon and thoracic surgeon. In all cases the tumor was resected from the brachial plexus using neurolysis while preserving the C-8 and T-1 nerve roots and lower trunk of the brachial plexus.

Results. One patient died 3 years posttreatment; the other four patients remain alive and well 2 to 5 years postoperatively. Hand function improved or remained normal in all four survivors, with postoperative intrinsic hand muscle function being Louisiana State University Medical Center Grade 5 in each patient. These results (2-year survival rate of 100\%) compare favorably with the Southwest Oncology Group Data (overall 2-year survival rate of 55\%; 70\% in patients who had undergone complete resection). With a minimum 2-year follow-up, $80 \%$ of patients remained alive and well, with normal hand function.

Conclusions. Although this patient series is small, the findings are extremely encouraging and suggest that the described treatment paradigm preserves survival as well as hand function in patients with Pancoast tumors.
\end{abstract}

KEY Words - brachial plexus - hand muscle • lung tumor • Pancoast tumor • superior sulcus tumor

$\mathrm{P}$ ANCOAST tumors, ${ }^{18,19}$ also known as apical lung tumors and superior pulmonary sulcus tumors, are bronchogenic cancers at the lung apex that invade structures located at the thoracic outlet, including the brachial plexus, sympathetic chain, subclavian vessels, ribs, and spine. This invasion produces symptoms and signs including shoulder, arm, and hand pain; hand wasting, weakness, and numbness; and Horner syndrome. Because of the lesion's peripheral location within the lung, neurological presentation usually occurs before respiratory symptoms develop. Untreated, Pancoast tumors are universally fatal, with death occurring within a matter of months from diagnosis. ${ }^{11,19}$

With the introduction of surgery, ${ }^{5}$ radiotherapy, ${ }^{27}$ and che-

Abbreviations used in this paper: LSUMC = Louisana State University Medical Center; SWOG = Southwest Oncology Group. motherapy ${ }^{23}$ the management of these tumors has evolved, and thus patient survival figures have improved significantly. With more aggressive cancer treatment, however, elements of the brachial plexus are frequently sacrificed, resulting in significant wasting and weakness in the hand. We aimed to determine whether a different approach, in which the brachial plexus and hand function are preserved, could be adopted without compromising patient survival.

\section{Clinical Material and Methods}

We reviewed data in all patients who had undergone combined thoracic-neurosurgical resection of apical lung tumors between January 2001 and December 2004. Our review followed the publication of the SWOG protocol for induction chemoradiotherapy prior to resection ${ }^{23}$ and enabled us to 
consider patients who had the opportunity to be treated according to that protocol. The evaluation of patients treated before December 2004 provided a minimum of 2 years of follow-up data. All patients had histologically confirmed bronchogenic apical lung tumors consistent with Pancoast syndrome. ${ }^{19}$

\section{Induction Chemotherapy and Radiotherapy}

After tumor diagnosis, induction chemotherapy and radiotherapy were begun. The chemotherapy regimen followed the SWOG protocol, ${ }^{23}$ which included at least two cycles of intravenously administered cisplatin and etoposide. A radiotherapy course of 45 Gy was administered in 25 fractions.

\section{Surgical Procedure}

Four to six weeks post-induction chemoradiotherapy, each patient proceeded to surgery. A thoracic surgeon and a neurosurgeon with experience in peripheral nerve surgery performed all operations. For the surgical approach, ${ }^{6,27,31}$ the patient is positioned in the lateral position, and a posterolateral thoracotomy is performed (Fig. 1). The approach, between the medial edge of the scapula and the spinous processes, can be extended superiorly to the neck, depending on the extent of the tumor. The thoracic surgeon exposes the lung apex and removes the posterior aspect of the first and second ribs. The neurosurgeon then dissects the T-1 nerve root, lower trunk of the brachial plexus, and C-8 nerve root. Whether this dissection proceeds from trunk to root, or root to trunk, depends on the morphological features of the tumor and the degree of nerve root involvement. If the nerve roots are buried in tumor or fibrous tissue, then it can be easier to start laterally with the lower trunk and dissect medially to expose the T-1 and C-8 nerve roots. If necessary, the C-7 nerve root and middle trunk can also be dissected. Once each neural element is identified, it is encircled with a Penrose drain and $360^{\circ}$ neurolysis is performed, enabling a clear plane to develop between the brachial plexus and the apical lung mass.

If the tumor is noted to extend into the intervertebral foramen on preoperative magnetic resonance imaging, then a hemilaminectomy is sometimes required to assist dissection of the tumor from the neural elements. For more extensive vertebral invasion, vertebral body resection and instrumented reconstruction are possible, ${ }^{8,32}$ but this procedure was not necessary among the current series of patients.

Following neurolysis of the C-7, C-8, and T-1 nerve roots and lower trunk of the brachial plexus and without fear of injuring the brachial plexus, the apical lung mass is then mobilized inferiorly by the thoracic surgeon. The apical lung mass is resected en bloc with the apical lung segment, upper lobe, or (rarely) the lung (Fig. 2). A biopsy sample of the hilar and mediastinal lymph nodes can be obtained as necessary.

The thoracotomy wound is closed over two chest tubes that are connected to underwater drains before the patient is transferred to the thoracic surgical high-dependency unit.

\section{Neurological Assessment}

A neurosurgeon performed an examination of brachial plexus function in all patients preoperatively and again at regular postoperative follow-ups. In addition to a routine

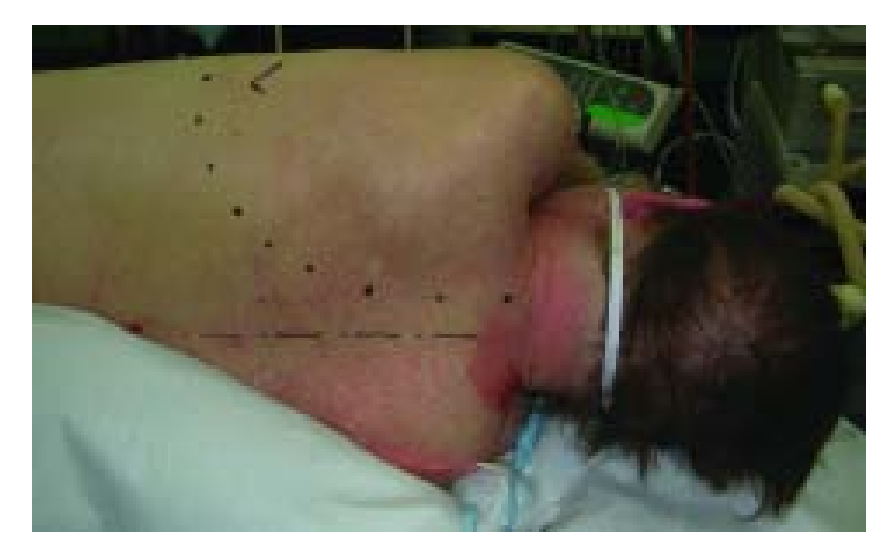

FIG. 1. Photograph depicting patient positioning for the combined thoracic-neurosurgical resection of a Pancoast tumor. Caret indicates the inferior angle of the left scapula; dashed and dotted line, the midline; dotted line, the skin incision.

neurological examination, particular notation was made regarding thenar and hypothenar wasting, and the following muscles were individually tested and their status recorded: abductor digiti minimi, abductor pollicis brevis, palmar interossei, dorsal interossei, and lumbrical muscles. Muscle and sensory function were graded according to the LSUMC system. ${ }^{13}$ The presence or absence of Horner syndrome was also recorded.

\section{Follow-Up Data}

Two end points were measured at the follow-up: hand function and patient survival at a minimum 2-year followup. Other demographic and clinical data collected included patient age and sex, presenting symptoms, duration of symptoms prior to diagnosis, hand dominance, side of tumor, histopathological findings, response to induction chemoradiotherapy, operative findings, operative complications, length of hospital stay, and time to last follow-up.

\section{Results}

Five patients with bronchogenic apical lung cancers and concomitant brachial plexus invasion underwent surgery between 2001 and 2004. All patients, one man and four women, had a history of smoking tobacco. The mean age at presentation was 57 years (range $40-71$ years; Table 1 ). The tumor in one patient was asymptomatic and diagnosed on a preoperative chest X-ray film obtained when she fractured her neck of the femur. The other four patients presented with symptoms typical of Pancoast syndrome, with varying degrees of shoulder, arm, and hand pain, and hand wasting, weakness, and numbness. One patient had Horner syndrome at presentation. The tumor was ipsilateral to the dominant hand in four patients. Chemoradiotherapy according to the SWOG protocol was completed in four patients. The patient in Case 4 was referred for induction chemotherapy and radiotherapy; however, she did not consent to radiotherapy and therefore underwent chemotherapy only. Postoperatively, histopathological data confirmed viable tumor in the tissue that was encasing the subclavian artery, and subsequently she agreed to a course of postoperative radiotherapy.

Operative findings are listed in Table 2. Varying degrees of compression and/or encasement of the T- 1 and C- 8 nerve 


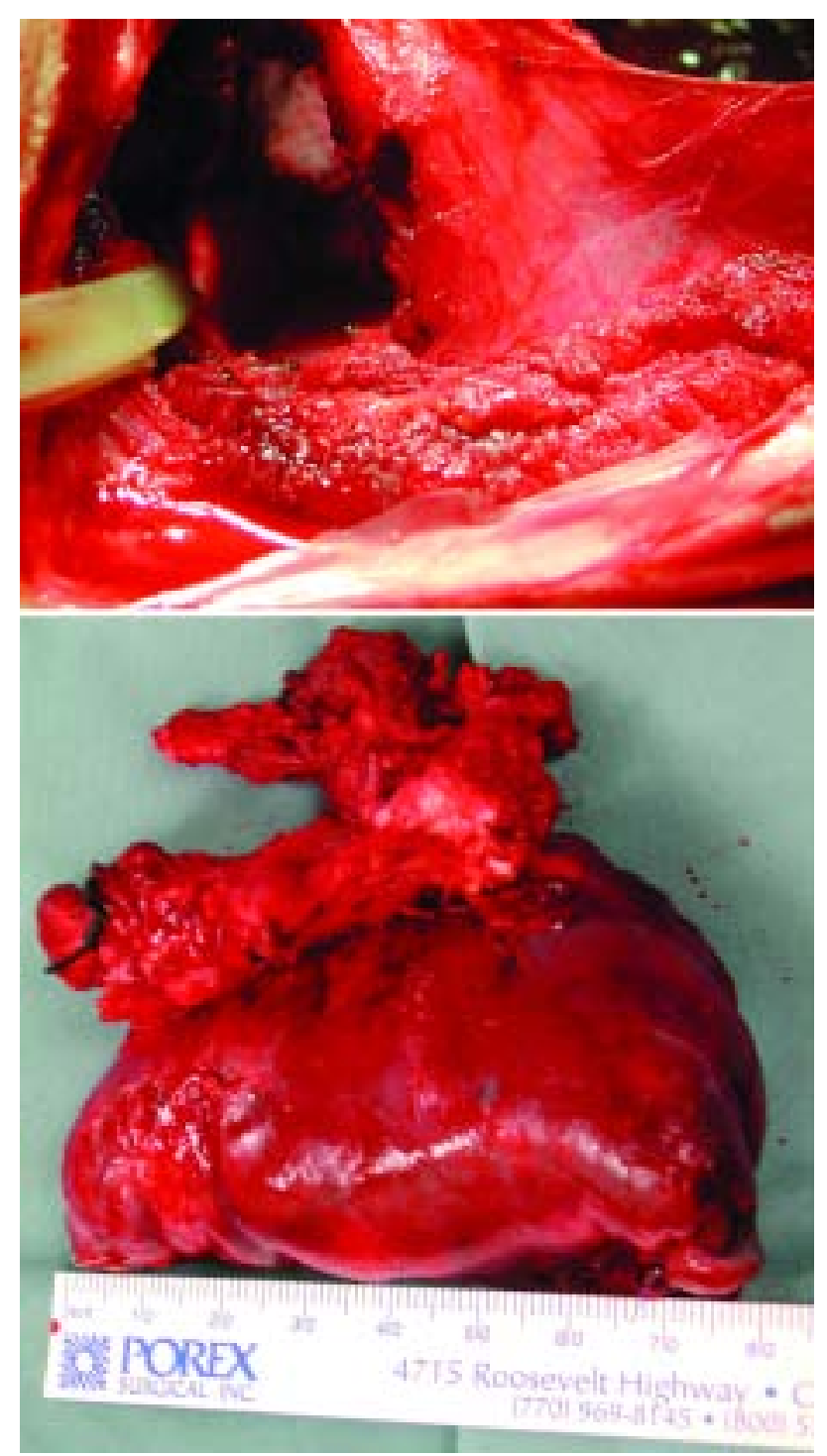

FIG. 2. Upper: Photograph illustrating the exposure after resection of the Pancoast tumor. A Penrose drain encircles the T-1 nerve root. Lower: Photograph showing the resected en bloc specimen. Note that the tumor and chest wall are attached to the lung apex.

roots were encountered. In all cases, after induction chemoradiotherapy, the tumor developed significant fibrosis, but this result did not prevent neurolysis of the neural elements in any case. In all patients, successful clearance of the macroscopic tumor was achieved, as was preservation of all brachial plexus elements. The only instance of incomplete resection occurred in the patient in Case 4, in whom the subclavian artery wall was invaded by tumor; it was determined that vascular resection and graft were not possible in this patient.

There was one surgical complication: a superficial wound infection developed in one patient, but it promptly responded to antibiotic treatment.

\section{Postoperative Hand Function}

The patients in Cases 1, 2, and 3 had intrinsic hand muscle function LSUMC Grade 3 or 4 preoperatively and im- proved to Grade 5 postoperatively. Hand function in the patient in Case 5 was Grade 5 both pre- and postoperatively. The patient in Case 4 presented with lumbrical muscle function of Grade 0 and other intrinsic hand muscle function of Grade 2; postoperatively, these grades did not change.

\section{Patient Survival}

Four patients remain alive and well; one patient (Case 4) died at 3 years postoperatively. Clinical and radiological follow-up assessment in all four survivors revealed no evidence of tumor recurrence. The duration of follow-up ranged from 2 to 5 years in all survivors (Table 2).

\section{Discussion}

\section{Anatomy of the Superior Sulcus}

The lung apex is situated within a region described as the thoracic inlet or the thoracic outlet, depending on one's perspective. This region is of great clinical importance because of the many structures running through or adjacent to the lung apex, including the subclavian vessels, the sympathetic trunk, the vertebral artery, the inferior brachial plexus, and the superior thoracic vertebra. Bronchogenic carcinomas arising in the apical or apicoposterior segments of the lung can directly invade any structure(s) within the thoracic inlet. Anatomically, it makes sense to refer to this location as the "lung apex" and therefore to describe lesions there as "apical lung tumors." However, when the presentation of an apical lung tumor involves the brachial plexus, sympathetic trunk, and vertebral body, then the clinical picture is that of a Pancoast tumor, and convention dictates the use of Pancoast's nomenclature, referring to these lesions as "superior pulmonary sulcus tumors." 19 In his original 1924 description of these lesions, Pancoast described them as "apical chest tumors"; 18 in his classic 1932 publication, however, he discarded that term in favor of "superior pulmonary sulcus tumor."'19 He preferred the latter term because he believed it implied "its approximate location and a lack of origin from the lung, pleura, ribs or mediastinum." He believed that these lesions arose from an embryonal epithelial rest and not from a primary lung tumor.

Confusion remains regarding the precise definition of the superior pulmonary sulcus. Paulson, ${ }^{20}$ a thoracic surgeon who has written extensively on Pancoast tumors, refers to Pancoast's original usage of the term superior pulmonary sulcus and then mistakenly reasons that this terminology refers to "the anatomical sulcus or groove made by the passage of the subclavian artery in the cupola of the pleura and apices of the upper lobes of the lungs." Teixeira ${ }^{29}$ discusses the widespread misunderstanding of the superior sulcus, refuting the claim that it is caused by a vascular impression in the lung. He defines this pulmonary sulcus as "the costovertebral gutter whose superior limit is the first rib arch and whose inferior limit is the insertion of the diaphragm in the thoracic cage." Therefore, the superior sulcus is the upper aspect of this gutter. It is apparent that this term is anatomically inappropriate. The fact that Pancoast tumors arise in the lung apex is sufficient justification to use the term "apical lung tumors," or, for those who prefer eponymics, "Pancoast tumor." The term superior sulcus is so widespread within the literature, however, that we fear it is now a permanent fixture. 
TABLE 1

Summary of presenting clinical features in five patients with bronchogenic apical lung cancers*

\begin{tabular}{|c|c|c|c|c|c|c|c|c|c|}
\hline $\begin{array}{l}\text { Case } \\
\text { No. }\end{array}$ & $\begin{array}{c}\text { Age } \\
(\text { yrs }) \\
\text { Sex }\end{array}$ & Presenting Symptom(s) & $\begin{array}{l}\text { Duration of } \\
\text { Symptoms } \\
\text { Before Diag- } \\
\text { nosis (mos) }\end{array}$ & $\begin{array}{l}\text { Hand Function } \\
\text { at Presentation } \dagger\end{array}$ & $\begin{array}{c}\text { Horner } \\
\text { Syndrome }\end{array}$ & $\begin{array}{l}\text { Hand } \\
\text { Domi- } \\
\text { nance }\end{array}$ & $\begin{array}{l}\text { Tumor } \\
\text { Side }\end{array}$ & $\begin{array}{c}\text { Tumor } \\
\text { Pathophysiology }\end{array}$ & $\begin{array}{l}\text { SWOG } \\
\text { Induction } \\
\text { Protocol } \\
\text { Completed }\end{array}$ \\
\hline 1 & $71, \mathrm{~F}$ & $\begin{array}{l}\text { cough, shoulder pain, } \\
\& \text { hand weakness }\end{array}$ & 24 & $\begin{array}{l}\text { C-7 Grade 4; C-8/T-1: } \\
\text { APB Grade 4, ADM } \\
\text { Grade 4; other intrinsic } \\
\text { hand muscles Grade 3; } \\
\text { sensation: Grade } 4\end{array}$ & no & $\mathrm{rt}$ & $\mathrm{rt}$ & NSCLC & yes \\
\hline 2 & $57, \mathrm{M}$ & shoulder pain & 9 & $\begin{array}{l}\text { all intrinsic hand muscles } \\
\text { Grade 4- }\end{array}$ & no & lt & $\mathrm{rt}$ & $\mathrm{SCC}$ & yes \\
\hline 3 & $40, \mathrm{~F}$ & arm pain \& weakness & 5 & $\begin{array}{l}\text { all intrinsic hand muscles } \\
\text { Grade } 4-\text {; sensation: T-1 } \\
\text { Grade } 4\end{array}$ & no & $\mathrm{rt}$ & $\mathrm{rt}$ & $\mathrm{LCC}$ & yes \\
\hline 4 & $54, \mathrm{~F}$ & $\begin{array}{l}\text { shoulder pain, hand } \\
\text { weakness, \& chronic } \\
\text { pain syndrome in hand; } \\
\text { attending chronic pain } \\
\text { clinic }\end{array}$ & ; & $\begin{array}{l}\text { wasting in intrinsic hand } \\
\text { muscles; finger abductor/ } \\
\text { adductor Grade } 2 \text {; lum- } \\
\text { bricals Grade 0; sensa- } \\
\text { tion: C-8 Grade 3, T1 } \\
\text { Grade } 1\end{array}$ & yes & lt & lt & adenocarcinoma & $\begin{array}{l}\text { chemotherapy } \\
\text { only }\end{array}$ \\
\hline 5 & $64, \mathrm{~F}$ & $\begin{array}{l}\text { lesion incidental finding } \\
\text { on presentation for } \\
\text { NOFF }\end{array}$ & NA & $\begin{array}{l}\text { all intrinsic hand muscles } \\
\text { Grade } 5\end{array}$ & no & $\mathrm{rt}$ & $\mathrm{rt}$ & NSCLC & yes \\
\hline
\end{tabular}

$*$ abductors = dorsal interossei muscles; adductors = palmar interossei muscles; $\mathrm{ADM}=$ abductor digiti minimi muscle; $\mathrm{APB}=$ abductor pollicis brevis muscle; $\mathrm{LCC}=$ large cell carcinoma; NA = not applicable; NOFF = neck of femur fracture; NSCLC = non-small cell lung carcinoma; SCC = squamous cell carcinoma.

$\dagger$ Grades based on the LSUMC scale.

\section{Anatomy of the Lower Trunk of the Brachial Plexus}

The brachial plexus forms from the anterior rami of the nerve roots of C-5, C-6, C-7, C-8, and T-1. The C-5 and C6 nerve roots join to form the upper trunk, the $\mathrm{C}-7$ nerve root continues as the middle trunk, and the nerve roots of C-8 and T-1 join to form the lower trunk. The T-1 nerve root runs over the pleural membrane, and T- 1 and the lower trunk are situated posterior to the subclavian artery.

Behind the clavicle, the lower trunk divides into two divisions. The posterior division joins the posterior cord, whereas the anterior division continues on as the medial cord of the brachial plexus. Branches from the medial cord include the medial pectoral nerve, medial cutaneous nerve of the arm, and medial cutaneous nerve of the forearm. The medial cord then divides into terminal branches: the ulnar nerve and the medial root of the median nerve. Medial cord motor fibers arising from C-8/T-1 and the lower trunk, which are destined to supply the intrinsic hand muscles, travel via the ulnar and median nerves, whereas the medial cord sensory fibers to the hand usually travel via the ulnar nerve. Interruption of the C-8/T-1/lower trunk results in wasting and weakness of intrinsic hand muscles, including the thenar (predominantly median nerve-innervated) and hypothenar (ulnar nerve-innervated) muscles, producing a clinical picture some refer to as the Gilliat-Sumner hand. ${ }^{9}$

\section{Anatomy of Sympathetic Control of the Eye}

From cells within the posterolateral hypothalamus, fibers run through the lateral brainstem to the intermediolateral gray matter (Rexed layer VII) of the T-1 spinal cord. Via the T-1 nerve root and the white communicating ramus, preganglionic fibers enter the sympathetic trunk and travel superiorly to the superior cervical ganglion. Postganglionic fibers then travel from the superior cervical ganglion along the internal carotid artery to the cavernous sinus, where the fibers join the ophthalmic division of the trigeminal nerve and the nasociliary nerve and then are distributed to the long ciliary nerves to supply the dilator pupillae., ${ }^{3,17}$ Other sympathetic fibers leave the internal carotid artery in the cavernous sinus to join the oculomotor nerve to supply the levator palpebrae muscle.

In their passage from the spinal cord to the superior cervical ganglion, these sympathetic fibers pass through the T1 rootlets and the stellate ganglion, and it is here that a Pancoast tumor can invade the structures, resulting in Horner syndrome, classically producing miosis, ptosis, enophthalmos, hyperemia of the eye, and hemifacial anhydrosis. (Although most physicians refer to this syndrome as "Horner syndrome," the French refer to it as "Claude Bernard syndrome," after the French physician who described the effects of the division of the cervical sympathetic trunk in $1853,{ }^{22} 16$ years before Johann Friedrich Horner's report. ${ }^{12}$ )

\section{Historical Background}

In 1838 Edward Hare, ${ }^{11}$ house surgeon of the Stafford County General Infirmary in England, detailed the first reported case of an apical lung tumor in a letter to the London Medical Gazette. He described a 40-year-old man with "pain, tingling and numbness along the course of the ulnar nerve of the left arm... pain through the left shoulder. . the pupil of the left eye became contracted; and the levator palpebrae ceased to perform its office." In addition to finding a palpable neck lump, Hare described the onset of neurological decline related to spinal cord compression and, ultimately, death 3 months after the onset of symptoms. Note the treatments used at the time: "leeches and blisters were repeatedly applied over the tumor, but without any effect. 
TABLE 2

Operative results and functional outcome in five patients who underwent Pancoast tumor resection

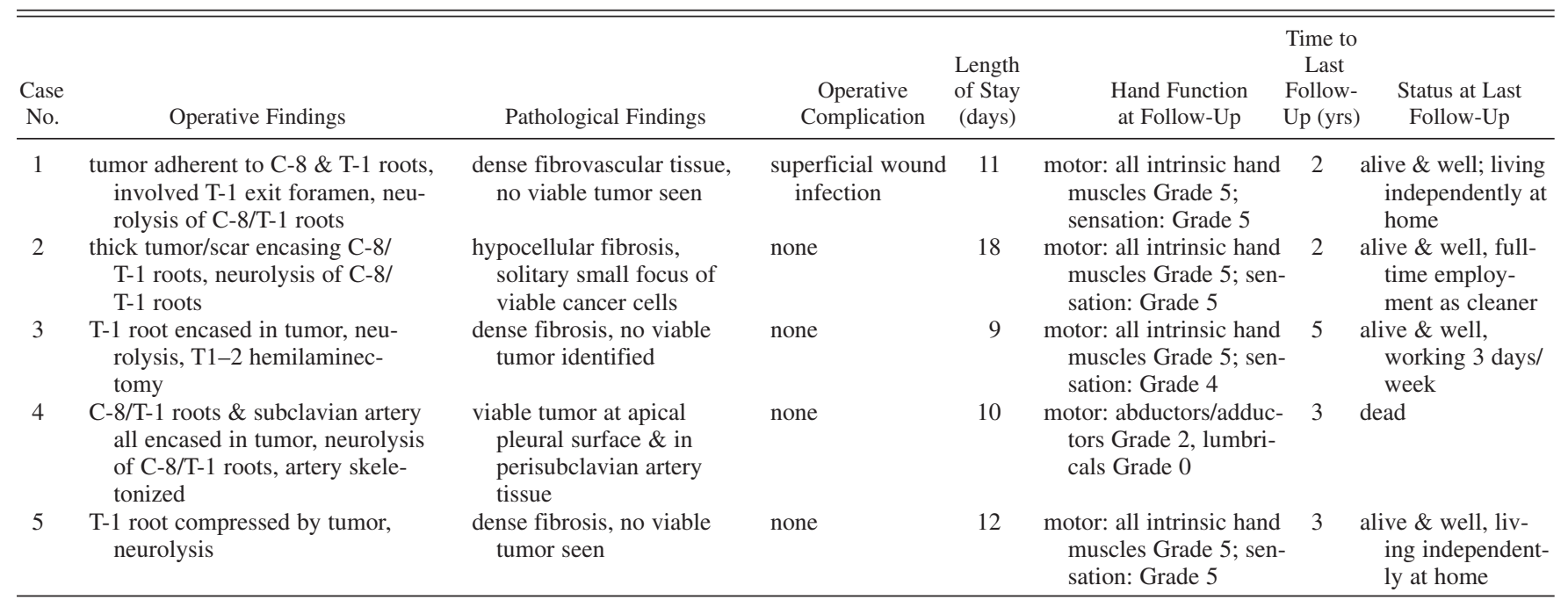

When the extract of belladonna was applied the pupil of the left eye recovered its natural size for a time." After describing the postmortem findings such as "the tumor lay upon the brachial plexus, being firmly attached to the spine at the origin of the third and fourth nerves of the plexus, both which were inseparable from it," Hare attempted to explain the clinical findings: "the connexion of the disease with the distress and paralysis along the course of the ulnar and median nerves is obvious, and is evidence in confirmation of the assertion that these nerves can be traced through the plexus to the last cervical and first dorsal nerve." He was unable to explain all, however: "The paralysis of the levator palpebrae, which receives a branch from the third pair; the contraction of the pupil. . . cannot be referred to any direct communication between the structural disease and these several affections. . . and is most frequently displayed in persons of a nervous temperament. . . and is parallel to what occurs in hysterical females, of which I have seen many examples."

The excellent understanding of the brachial plexus and median and ulnar nerve anatomy is overshadowed by Hare's lack of knowledge regarding the sympathetic supply to the eye, and it is this failure to understand the patient's physical signs that prevented him from being credited with the eponymous title. Note, however, that Hare never had the opportunity to pursue the sympathetic supply to the eye any further, because he died of typhus the day before his letter was published.22

In 1924 Pancoast,,$^{18}$ a radiologist from Philadelphia, published his first paper in which three cases of apical chest tumors were reported. A subsequent and more notable paper was his chairman's address that was read before the Section on Radiology at the American Medical Association meeting in New Orleans in $1932 .{ }^{19}$ At that time, Pancoast had adopted the name "superior pulmonary sulcus tumor." He reported on seven cases with "clinical and roentgenographic characteristics which would warrant the inclusion of the tumors in a group which could be recognised as a pathologic entity." Pancoast listed the clinical features as "pain around the shoulder and down the inner side of the arm, and often the ulnar side of the forearm, loss of power and wasting of the muscles of the hand, Horner's syndrome and signs mainly of dullness in the apex of the chest." The radiological features were "small and circumscribed shadow in the apex due to lung displacement, and destruction of the posterior portions of one or more ribs and the adjacent articular and transverse processes and possibly a little of the sides of the bodies of one or more vertebrae."

Pancoast stated that Horner syndrome is "an essential manifestation," as it occurred in all seven of his reported cases, whereas hand weakness or wasting occurred in only three cases. Unlike Hare before him, Pancoast succeeded in correlating the clinical and radiological findings to describe this disease entity, although he did err in his pathological assessment. He believed that these tumors took "origin in an embryonal epithelial rest" and that "one can practically rule out primary lung cancer." With regard to prognosis, Pancoast noted that these tumors were rapidly fatal, with death occurring 11 to 14 months after symptom onset. He believed that these lesions were not surgically treatable, with radiation treatment being the only possible option.

In the 15 to 20 years after Pancoast's publication, additional reports of this fatal tumor appeared. In 1956, however, Chardack and MacCallum, ${ }^{5}$ published the first report of resection combined with postoperative radiation treatment and a 5-year survival. (The patient survived 5 years and 10 months after surgery and died of bronchopneumonia. Postmortem examination showed no evidence of tumor recurrence or metastases.) Chardack and MacCallum described the en bloc resection of the C-8 and T-1 nerve roots and stated that at the follow-up "neurological examination revealed the expected deficit due to the removal of roots."

In 1961 Shaw and colleagues described a series of $18 \mathrm{pa}-$ tients who had been treated with a combination of preoperative radiation and resection 1 month following completion of radiation therapy. ${ }^{27}$ Although the follow-up period was less than 12 months for nine of the 18 patients, the overall result appeared promising, given that the disease had previously had a universally fatal outcome. Encouraged by these results, Paulson ${ }^{20}$ continued this therapeutic protocol (preoperative radiotherapy followed by surgery 3 to 6 weeks 
later) and reported on a series of 61 patients treated between 1956 and 1973 . He found a survival rate of $34 \%$ at 5 years and $29 \%$ at 10 years. He proposed that the rationale for preoperative radiotherapy was "to modify the extent of the disease so that the lesion is better localised and thus more completely resectable, with improved results but without increased morbidity." Based on these findings, the combined radiotherapy and surgery protocol remained the standard treatment for Pancoast tumors until the late 1990s. During this time, the results of treatment for Stage IIIA non-small cell lung cancer using combined induction chemoradiotherapy and surgery were found to be better than those of radiotherapy and surgery. This finding stimulated the use of induction chemoradiation and resection by the SWOG in Pancoast tumors and the publication of Phase II data in $2001 .^{23}$ The SWOG data showed a 2-year survival rate of $55 \%$ for all eligible patients and $70 \%$ for those who had undergone complete resection.

From Hare's time, when the Pancoast tumor was described but not understood, and Pancoast's time, when the clinical and radiological signs were understood but no treatment was available, through to today, when induction chemoradiation and resection offer prolonged survival, an essential element of these tumors has not been adequately addressed: What does one do with the brachial plexus to preserve hand function?

\section{Management of the Brachial Plexus and Preservation of Hand Function}

Understandably, the primary goal in the management of Pancoast tumors has hitherto been to improve patient survival. As mentioned previously, survival figures for Pancoast tumors have evolved from 3 months in Hare's patient in $1838^{11}$ to a 2 -year survival rate of $70 \%$ in the SWOG series in $2001 .{ }^{23}$ During this time, how have the brachial plexus and hand function fared?

Hare obviously understood the anatomy and function of the brachial plexus; consider his postmortem description of brachial plexus involvement and his acknowledgment that the involvement of the lower roots of the brachial plexus accounted for "paralysis along the course of the ulnar and median nerves." In his classic paper, Pancoast detailed the pathological entity that bears his name, but he stated that the muscular wasting corresponded "to an ulnar supply from the eighth cervical and first thoracic nerves." 19 This assertion ignored the median nerve supply to the hand, derived from the C-8 and T-1 nerve roots. It is not apparent from Pancoast's descriptions whether any of his patients had complete C-8/T-1 loss, and thus loss in the median and ulnar territories, or whether the absence of any documentation regarding median nerve loss was deliberate on his part.

Chardack and MacCallum ${ }^{5}$ resected the C-8 and T-1 nerve roots in their en bloc excisions, accepting the neurological deficit in the hand as the cost of prolonging patient survival. In blunt terms, Shaw and colleagues ${ }^{27}$ stated that "none of the structures usually found to be invaded are necessary to the economy of the body and therefore can be sacrificed. Ribs, intercostal structures, sympathetic nerves, brachial plexus roots.... The fact that permanent neurologic defects may result is of little consequence." Similarly, according to his 1975 paper, ${ }^{20}$ Paulson routinely resected the lower trunk of the brachial plexus, stating "permanent neu- rological defects resulting from resection of the lower trunk of the brachial plexus involve the ulnar nerve, but are not incapacitating." He also stated "contraindications to operation include extensive invasion of the brachial plexus." This statement is consistent with the standard of the time and obviously is no longer valid today.

In 1987 Shahian et al. ${ }^{26}$ performed surgery in 18 patients after preoperative radiotherapy. Preoperatively, "ulnar neuropathy was present in varying degrees." Again, this assertion ignores the true anatomical nature of the lower trunk of the brachial plexus. The neuropathy is a radiculopathy (for example, T-1 with or without C-8), a trunk lesion, or both. It is not an ulnar neuropathy, given that almost certainly there is also a degree of median nerve dysfunction from the C-8/T-1 supply. In 11 patients, "the inferior root of the brachial plexus was intentionally sacrificed." The use of the term "inferior root" is somewhat misleading; does it refer to the T-1 root, the C-8 root (the most inferior of the cervical roots of the plexus), or the inferior trunk? Nevertheless, Shahian and colleagues were the first group to publish functional results in 10 long-term survivors. They graded pain relief, shoulder mobility, and neurological dysfunction, and using the scale excellent, good, fair, and poor, they reported the following results. Pain relief was excellent in $80 \%$, good in $10 \%$, and poor in $10 \%$; shoulder mobility was excellent in $70 \%$, good in $10 \%$, and fair in $20 \%$; and neurological function was excellent in $30 \%$, good in $60 \%$, and fair in $10 \%$. Unfortunately, the authors did not define the terms "excellent," "good," "fair," or "poor," so it is difficult to glean much information from these figures.

Sundaresan and associates ${ }^{28}$ described a series of 30 patients with Pancoast tumors, advocating a team approach, that is, the combined efforts of a thoracic surgeon and a neurosurgeon. Their study included a heterogeneous group of patients, with some having undergone preoperative radiotherapy and/or chemotherapy and others having no preoperative treatment. Some patients had significant spinal involvement, whereas others did not. One of the few constant elements in their series was the resection of the T-1 root in all patients. In four patients, the C-8 root was also sacrificed. Unfortunately, except for a comment that all patients experienced pain relief, results of neurological functional outcome were not presented.

In his 1988 review of Pancoast tumors, Urschel ${ }^{30}$ advocated resection of the lower trunk of the brachial plexus. In their 1997 review, Arcasoy and Jett ${ }^{1}$ stated that surgical treatment "may also be accompanied by resection of... lower trunks of the brachial plexus." (We assume that the plural "trunks" is an error in their paper and that they really meant the lower trunk, or the lower two roots.) No results on hand function were presented.

In a 1999 review of nine patients with Pancoast tumors that had invaded the vertebral column, York et al..$^{32}$ described having operated on tumors with a much greater degree of spinal invasion than occurred in our series. With regard to the brachial plexus, they stated as follows: "an attempt is made to spare the T1 nerve root as it crosses beneath the angle of the first rib to join the C8 nerve root." Despite this intention, the T-1 nerve root was sacrificed in seven of the nine patients who underwent surgery. Regarding hand function and grading, the authors presented their results according to the following descriptions: intact, weak, weaker, decreased sensation, and unchanged. Of the seven 
patients with a sectioned $\mathrm{T}-1$ root (one patient also had a sectioned C-8 root), five patients demonstrated more weakness, one patient had decreased sensation, and one patient was intact. In another paper that year, Gandhi and associates $^{8}$ described 17 patients requiring resection of Pancoast tumors with vertebral involvement. In all patients, nerve roots were resected, but the anatomical levels of the roots were not described. No results on hand function were presented; however, the authors did note that six of six patients with positive margins at the vertebral body, brachial plexus, and paraspinous region had local recurrence and died within 2 years of treatment, whereas one of 11 patients with negative margins experienced a recurrence. The 2-year survival rate in the negative-margin group was $80 \%$. This rate can be compared with that in the present study, in which we did not measure margins at the level of the brachial plexus because the plexus was preserved with neurolysis alone. The fact that all patients in the present study survived beyond 2 years makes an interesting comparison.

In a seminal paper published in $2001,{ }^{23}$ detailing the results of the first prospective multiinstitutional trial on Pancoast tumors, the SWOG data contain no mention of brachial plexus or hand function at all. Similarly, in a review of 139 patients treated in seven centers in France, there was no mention of brachial plexus management or functional hand outcome..$^{15}$ Grunenwald and colleagues ${ }^{10}$ reviewed 19 patients with lung cancer invading the spine. They routinely resected involved nerve roots. Hand and brachial plexus functional results were not presented.

In 2002 Bilsky et al. ${ }^{4}$ reviewed data from a large series of patients who underwent surgery for superior sulcus tumors. Of the 187 patients, 21 had brachial plexus involvement. The T-1 nerve root was involved in 17 patients, and the C-8/lower trunk was involved (in addition to the T-1 nerve root) in four patients. The authors reported that they routinely resect the $\mathrm{T}-1$ nerve root but not the C-8 nerve root. They described the use of biopsy samples of the C-8 nerve root and lower trunk but they did not report whether the C-8 nerve root is actually sacrificed if the biopsy is positive. With regard to hand function following T-1 nerve root resection, the authors stated that "generally, this results in mild motor dysfunction of the hand intrinsic muscles that gradually improves." Specific details regarding hand function were not presented. Interestingly, Bilsky et al. ${ }^{4}$ have stated that "in our opinion, involvement of the $\mathrm{C} 8$ nerve root or lower trunk of the plexus is considered a contraindication to surgery unless a significant preoperative deficit exists or possibly the non-dominant hand is involved." As can be seen from our results, this statement is no longer applicable. Even with involvement of the C-8 nerve root and minimal preoperative deficit, neurolysis with preservation of brachial plexus elements and hand function can definitely be achieved.

In his 2003 review of Pancoast tumors, Detterbeck ${ }^{7}$ did not provide any details regarding hand function posttreatment and made some bold statements regarding brachial plexus management, including the following: "extensive involvement of the brachial plexus remains a contraindication to resection," and "it may be reasonable to explore the role of the more extensive surgical procedure such as a forequarter amputation." Obviously, we do not support these opinions in any manner whatsoever, and we have shown that brachial plexus involvement is not a contraindication to re- section and that hand function can be preserved by neurolysis at the time of surgery (when used in conjunction with induction chemoradiation treatment).

Mazel and colleagues ${ }^{16}$ reviewed a series of 36 patients with extensive tumors invading the spine, who had undergone radical resection and spinal reconstruction. The reported neurological outcomes related to spinal cord function (Frankel grade) and not to brachial plexus function. These authors reported that in most cases the T-1 nerve root was resected, but based on their discussion it is not apparent whether they always preserved the $\mathrm{C}-8$ nerve root.

\section{Preserving Hand Function and Patient Survival}

The emphasis on survival figures has dominated most recent publications on the management of Pancoast tumors. ${ }^{1,2}$, 4,7,8,10,14-16,21,23-25,32 The aggressive oncological surgical dictum of "clear resection margins" has been the driving principle in most surgical studies. As mentioned earlier, Gandhi et al. ${ }^{8}$ reported that in patients with positive margins, six of six patients had a local recurrence, compared with only one patient among 11 with negative margins. Furthermore, no patient with positive margins survived more than 2 years after treatment. Note, however, that their treatment protocol consisted of preoperative radiotherapy alone and not chemotherapy.

Our treatment protocol differed from that regimen in two ways. First, induction chemoradiotherapy before surgery had a profound effect on tumor size, tumor burden, and patient survival. The histopathological finding of dense fibrosis in all resected specimens was evidence of the profound tumoricidal effect of induction chemoradiotherapy. Second, we aimed to preserve brachial plexus function in all cases. We were able to do this because the induction chemoradiotherapy has significant tumoricidal action and because the team approach of an experienced peripheral nerve surgeon working with a thoracic surgeon brings the perspective of each to the operating room. Following induction chemoradiotherapy, the thoracic surgeon's objective is to deliver the lung apex and chest wall in an en bloc fashion; this part of surgery is the cancer operation. The peripheral nerve surgeon performs extensive neurolysis of the roots and lower trunk of the brachial plexus to remove the fibrotic mass that has developed during the chemoradiation therapy; this portion of the surgery is the nerve decompression operation.

Although our sample is small, our results are very encouraging because they satisfy both objectives: 1) patient survival and 2) preservation of hand function. In four of five patients, intrinsic hand muscle function remained or improved to LSUMC Grade 5, and all four patients remain alive and progression free 2 to 5 years after resection. The one patient death (Case 4) occurred in a woman who had a very delayed presentation with tumor invasion into both the brachial plexus and the subclavian artery. Note also that she was given induction chemotherapy but not induction radiotherapy. Radiotherapy was administered postoperatively. Although we have included her data in our calculations based on an intention to treat, it must be emphasized that she did not complete the preoperative induction treatment, consenting to preoperative chemotherapy only. One can only speculate whether completion of preoperative radiotherapy would have altered her clinical course given the extensive tumor invasion into the brachial plexus and subclavian ar- 
tery and her very delayed presentation more than 1 year after symptom onset.

Of the patients who completed induction chemoradiotherapy before surgery, there was universal preservation of hand function, and all remained alive and well at a minimum 2-year follow-up. Although this series is small, our results have encouraged us to continue with this treatment paradigm and should encourage other centers to aim for both patient survival and hand function preservation.

The principles adopted in the featured surgical technique have been used in other patients with nonbronchogenic apical tumors. For example, a 24-year-old professional guitar player with neurofibromatosis Type 1 who had presented with a cough was found to have interstitial lung disease and a right apical neurofibroma compressing the C-8/T-1 nerve roots with Grade 4 weakness in the right intrinsic hand muscles. Resection of the tumor and lung apex via a posterior thoracotomy together with preservation of the C-8 and T-1 nerve roots resulted in complete recovery of intrinsic hand muscle function and a subsequent return to guitar playing. The combined peripheral nerve surgeon/thoracic surgeon approach to dealing with this tumor provided the best longterm outcome for preservation of hand function.

\section{Conclusions}

The management of Pancoast tumors has undergone radical evolution since Hare's original description of these lesions in 1838. With induction chemoradiotherapy and surgery providing excellent survival results in many patients hitherto considered untreatable, the time has arrived to abandon the notion of acceptable neurological deficits and to aim for the preservation of hand function in all patients. Although our patient series is small, our findings are extremely encouraging and suggest that our treatment paradigm preserves not only survival but also hand function in patients with Pancoast tumors.

\section{References}

1. Arcasoy SM, Jett JR: Superior pulmonary sulcus tumors and Pancoast's syndrome. New Engl J Med 337:1370-1376, 1997

2. Archie VC, Thomas CR Jr: Superior sulcus tumors: a minireview. Oncologist 9:550-555, 2004

3. Barr ML, Kiernan JA: The Human Nervous System. An Anatomical Viewpoint, ed 6. Philadelphia: JB Lippincott, 1993, pp 364-371

4. Bilsky MH, Vitaz TW, Boland PJ, Bains MS, Rajaraman V, Rusch VW: Surgical treatment of superior sulcus tumors with spinal and brachial plexus involvement. J Neurosurg 97 (3 Suppl):301-309, 2002

5. Chardack WM, MacCallum JD: Pancoast tumor; five-year survival without recurrence or metastases following radical resection and postoperative irradiation. J Thorac Surg 31:535-542, 1956

6. Davis GA, Knight S: Brachial plexus surgery and apical lung tumors. Cancer Forum 28:11-12, 2004

7. Detterbeck FC: Changes in the treatment of Pancoast tumors. Ann Thorac Surg 75:1990-1997, 2003

8. Gandhi S, Walsh GL, Komaki R, Gokaslan ZL, Nesbitt JC, Putnam JB, et al: A multidisciplinary surgical approach to superior sulcus tumors with vertebral invasion. Ann Thorac Surg 68: 1778-1784, 1999

9. Gilliatt RW, Le Quesne PM, Logue V, Sumner AJ: Wasting of the hand associated with a cervical rib or band. J Neurol Neurosurg Psychiatry 33:615-624, 1970

10. Grunenwald DH, Mazel C, Girard P, Veronesi G, Spaggiari L,
Gossot D, et al: Radical en bloc resection for lung cancer invading the spine. J Thorac Cardiovasc Surg 123:271-279, 2002

11. Hare E: Tumor involving certain nerves. London Med Gaz 1: $16-18,1838$

12. Horner JF: Uber eine Form von Ptosis. Klinische Monatsblatter fur Augenheilkunde 7:193-198, 1869

13. Kline DG, Hudson AR: Nerve Injuries. Operative Results for Major Nerve Injuries, Entrapments, and Tumors. Philadelphia: WB Saunders, 1995, pp 561-653

14. Kraut MJ, Vallieres E, Thomas CR Jr: Pancoast (superior sulcus) neoplasms. Curr Probl Cancer 27:81-104, 2003

15. Martinod E, D'Audiffret A, Thomas P, Wurtz AJ, Dahan M, Riquet M, et al: Management of superior sulcus tumors: experience with 139 cases treated by surgical resection. Ann Thorac Surg 73:1534-1540, 2002

16. Mazel C, Grunenwald D, Laudrin P, Marmorat JL: Radical excision in the management of thoracic and cervicothoracic tumors involving the spine: results in a series of 36 cases. Spine 28:782-792, 2003

17. McMinn RMH: Last's Anatomy: Regional and Applied, ed 8. London: Churchill Livingstone, 1990, pp 510-516

18. Pancoast HK: Importance of careful roentgenray investigations of apical chest tumors. JAMA 83:1407-1411, 1924

19. Pancoast HK: Superior pulmonary sulcus tumor. JAMA 99: 1391-1396, 1932

20. Paulson DL: Carcinomas in the superior pulmonary sulcus. J Thorac Cardiovasc Surg 70:1095-1104, 1975

21. Pitz CC, de la Riviere AB, van Swieten HA, Duurkens VAM, Lammers JW, van den Bosch JM: Surgical treatment of Pancoast tumors. Eur J Cardiothorac Surg 26:202-208, 2004

22. Ross IB: The role of Claude Bernard and others in the discovery of Horner's syndrome. J Amer Coll Surg 199:976-980, 2004

23. Rusch VW, Giroux DJ, Kraut MJ, Crowley J, Hazuka M, Johnson D, et al: Induction chemoradiation and surgical resection for non-small cell lung carcinomas of the superior sulcus: intitial results of Southwest Oncology Group trial 9416 (Intergroup trial 0160). J Thorac Cardiovasc Surg 121:472-483, 2001

24. Rusch VW, Parekh KR, Leon L, Venkatraman E, Bains M, Downey RJ, et al: Factors determining outcome after surgical resection of T3 and T4 lung cancers of the superior sulcus. J Thorac Cardiovasc Surg 119:1147-1153, 2000

25. Shahian DM: Contemporary management of superior pulmonary sulcus (Pancoast) lung tumors. Curr Opin Pulm Med 9: 327-331, 2003

26. Shahian DM, Neptune WB, Ellis FH: Pancoast tumors: improved survival with preoperative and postoperative radiotherapy. Ann Thorac Surg 43:32-38, 1987

27. Shaw RR, Paulson DL, Kee JL Jr: Treatment of the superior sulcus tumor by irradation followed by resection. Ann Surg 154: 29-40, 1961

28. Sundaresan N, Hilaris BS, Martini N: The combined neurosurgical-thoracic management of superior sulcus tumors. J Clin Oncol 5:1739-1745, 1987

29. Teixeira JP: Concerning the Pancoast tumor: What is the superior pulmonary sulcus? Ann Thorac Surg 35:577-578, 1983

30. Urschel HC Jr: Superior pulmonary sulcus carcinoma. Surg Clin North Am 68:497-509, 1988

31. Vallieres E, Karmy-Jones R, Mulligan MS, Wood DE: Pancoast tumors. Curr Probl Surg 38:306-376, 2001

32. York JE, Walsh GL, Lang FF, Putnam JB, McCutcheon IE, Swisher SG, et al: Combined chest wall resection with vertebrectomy and spinal reconstruction for the treatment of Pancoast tumors. J Neurosurg (1 Suppl) 91:74-80, 1999

Manuscript received February 15, 2007.

Accepted in final form April 24, 2007.

Address reprint requests to: Gavin A. Davis, F.R.A.C.S., Suite 53 Neurosurgery, Cabrini Medical Centre, Malvern, Victoria 3144, Australia.email: gadavis@ netspace.net.au. 